Sādhanā Vol. 36, Part 1, February 2011, pp. 125-139. @ Indian Academy of Sciences

\title{
Intelligent detection of microcalcification from digitized mammograms
}

\author{
SARWESH PARADKAR* and S S PANDE \\ Computer Aided Manufacturing Laboratory, Mechanical Engineering Department, \\ Indian Institute of Technology Bombay, Powai, Mumbai 400 076, India \\ e-mail: sarwesh_p@iitb.ac.in; s.s.pande@iitb.ac.in
}

MS received 20 October 2009; revised 27 September 2010; accepted 6 December 2010

\begin{abstract}
This paper reports the design and implementation of an intelligent system for detection of microcalcification from digital mammograms. A neuron based thresholding strategy has been developed to reduce the number of candidate pixels. A back propagation neural network (BPNN) classifier has been used to classify the pixels into positive (affected) and normal ones. The false positives generated in the process are eliminated using the connected component analysis and the elongated component removal algorithms in succession. Suspected areas of microcalcification are detected and marked on the mammogram. The system was rigorously tested for the available images and was found to be quite robust, consistent and fast in detection. The output image with prompts generated by the system can form an important input to a radiologist for the final diagnosis.
\end{abstract}

Keywords. Breast cancer; digital mammogram; artificial neural networks; microcalcification detection.

\section{Introduction}

Breast cancer is the most frequently diagnosed form of cancer in women worldwide with about one million cases reported every year. It represents over $20 \%$ of all malignancies in females (Parkin et al 2001). About 50\% of high risk cases are found to occur in the developed world. National Cancer Registry Project (2001) has reported an increasing trend in the occurrence of breast cancer cases in India.

It has been observed that localized cancer leads to a five year survival rate of $97.5 \%$, compared to only $20 \%$ survival rate for cancer that has spread to distant organs (Jemal et al 2004). Early detection of breast cancer is therefore, of utmost importance. Self-examination of breast is not adequate for the early detection of breast cancer. There is no evidence of reduction in mortality rates.

*For correspondence 
Today, mammography is the best available method for early detection of breast cancer. It has been shown to be very effective in the reduction of breast cancer mortality rates. However interpreting screening mammograms is not easy as the sensitivity of mammography is affected by the quality of the image and the level of expertise of the radiologist. False positive cases have led to unnecessary biopsies, chemotherapy and the psychological trauma associated with it (Rangayyan et al 2007).

Computer Aided Diagnosis systems (CAD) could offer a cost effective alternative to double reading of the mammograms and can prompt the radiologist to view suspected areas for reducing errors in diagnosis. The CAD system could reduce the chances of false detection of positives/negatives and help to add expertise to the decision of a relatively inexperienced radiologist. The diagnosis could, in effect, be a joint decision based on data processing results given by the CAD system and the experience of the doctor. The commercially available computer aided diagnosis systems (CAD) being used by the doctors are, however, quite primitive in nature. They do not have any automatic detection capability but merely provide facilities such as image contrasting, zooming and marking of high intensity regions. These systems need expert radiologists to interpret the mammograms and diagnose.

Researchers and medical equipment developers worldwide are, thus, attempting to develop robust and efficient $\mathrm{CAD}$ systems for the automatic detection of micro calcifications from radiological images. The work reported in the present paper is an attempt to develop such a system.

\section{Literature review}

Literature reports the attempts made by the researchers worldwide to develop techniques for automated processing of digital radiological images for mammography. Important among them include neural networks (Delogu et al 2007; Halkiotis et al 2007; Wei et al 2009; Oh et al 2009), genetic algorithms (Jiang et al 2007), wavelet based techniques (Laine et al 1994) and multifractal segmentation based techniques (Stojic et al 2006). Neural network based techniques are found to be most promising among these reported approaches for pattern recognition.

The digital mammograms often have a lot of variation due to poor image quality. This variability (imprecise data) is handled very efficiently by a neural network through its supervised learning ability for pattern recognition. The accuracy of a neural network can be improved further by including larger training sets. The neural network based techniques are therefore, getting popular for the pattern recognition applications. Very few attempts have however, been reported on the use of neural networks for detection of micro calcifications (positives) from the digital mammograms. Halkiotis et al (2007) used a segmentation technique to extract the mass from the region and compute features based on the size and shape of the lesion. A neural network classifier merges the features to predict likelihood of malignancy for that region. Wei et al (2009) had used a two view method in which true positive and false positive results from two views are compared and a weighted score is computed. The results from the two views along with the weighted scores act as inputs to the neural network for the final result. The above methods rely predominantly on the calculation of a feature set for the image pixels followed by the classification as affected or normal. These methods are computationally intensive due to the lack of pre-processing of the image. Oh et al (2009) illustrated the use of a multi module system in which the region of interest is first segmented using contrast property of the grey level co-occurrence matrix (GLCM). The potential microcalcification pixels are then detected by foveal method and finally false positives are eliminated 
using a set of 8 statistical features. This technique, however, results in the enhancement of breast structures such as dense tissues, ducts and skin folds which have intensities similar to micro calcification. As a result elimination of false positives becomes difficult. Additional features need to be considered for better detection performance. Pal et al (2008) have reported a set of statistical features to be computed for each image pixel. These features are merged and processed by a neural network to classify the said pixel to be affected or normal.

Literature thus suggests that there is a need to develop a robust and efficient computer aided diagnosis (CAD) system for automatic detection of micro calcification from digital mammograms which will be reasonably fast and accurate for real life applications.

In this paper, we present the development of a PC-based system for the intelligent detection of microcalcification from digital mammograms using an artificial neural network. Various design, implementation and testing issues of the system are presented in details, in the sections to follow.

\section{System overview}

Figure 1 shows the modular architecture of the system developed by us. It primarily comprises of three functional modules - pre-processing of digital mammogram, neural network based classification and the elimination of false positives for diagnosis.

Functional working of these modules and the associated algorithms are presented in the sections to follow.

\subsection{Pre-processing of the digital image}

The digital mammogram image in the jpeg format is input to the system. The GUI has been designed similar to the existing CAD systems so that the radiologists can feel familiar. Commercial systems being used by doctors in India have features like image negation, zoom and contrast enhancement. We retained these features in our system. The physician can have a better view of the mammogram using these familiar features before going for the automatic detection. For the development and testing of the system, several digital mammograms of both normal as well as affected patients were chosen from M/s Tata Memorial Hospital in consultation with the radiologists. In addition, images from the Essex University website were also used.

The digital image input to the system is pre-processed to filter out low intensity pixels which are not candidates for microcalcification. This will save the valuable processing time. To explain the working of the various functional modules of our system, we have taken a portion $(512 \times$ 512 pixel size) out of the region of interest in a typically affected image.

3.1a Plain thresholding: The digitized mammogram contains a large number of background pixels apart from the actual region of interest. Lot of computing effort and processing time will be unnecessarily spent if such an image is directly used. Microcalcifications are high intensity occurrences on a digitized mammogram. We can therefore, set an intensity threshold and filter out the pixels which are not the candidate for microcalcification. After studying the various available affected images, we have set this threshold to a gray level intensity of 100 in our work. Pixels having gray level intensity higher than 100 are retained for further processing. Figures $2 \mathrm{a}$ and $\mathrm{b}$ show a digitised mammogram and its corresponding image obtained after the first (plain) thresholding operation. The original image comprised of 250000 candidate pixels was reduced to 140026 after the thresholding operation. 


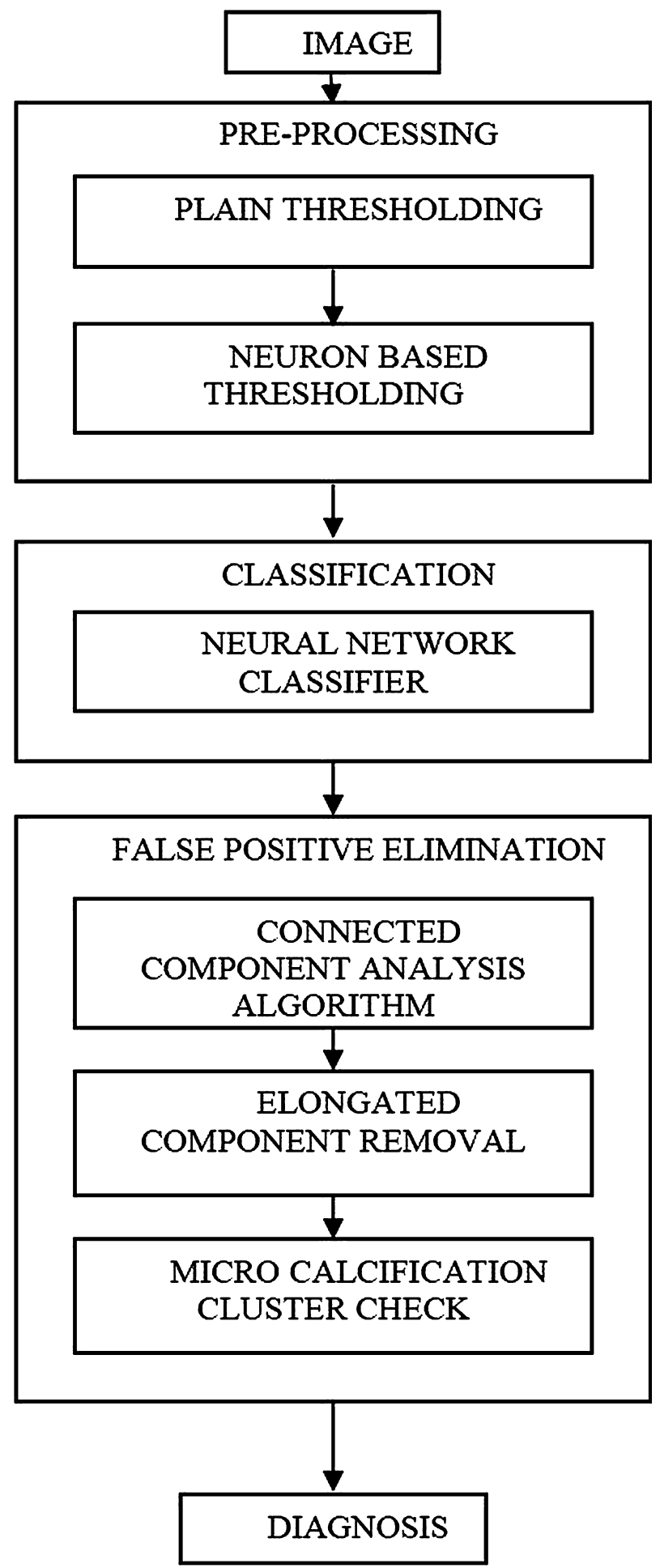

Figure 1. Modular diagram of the system. 


\section{(a)}

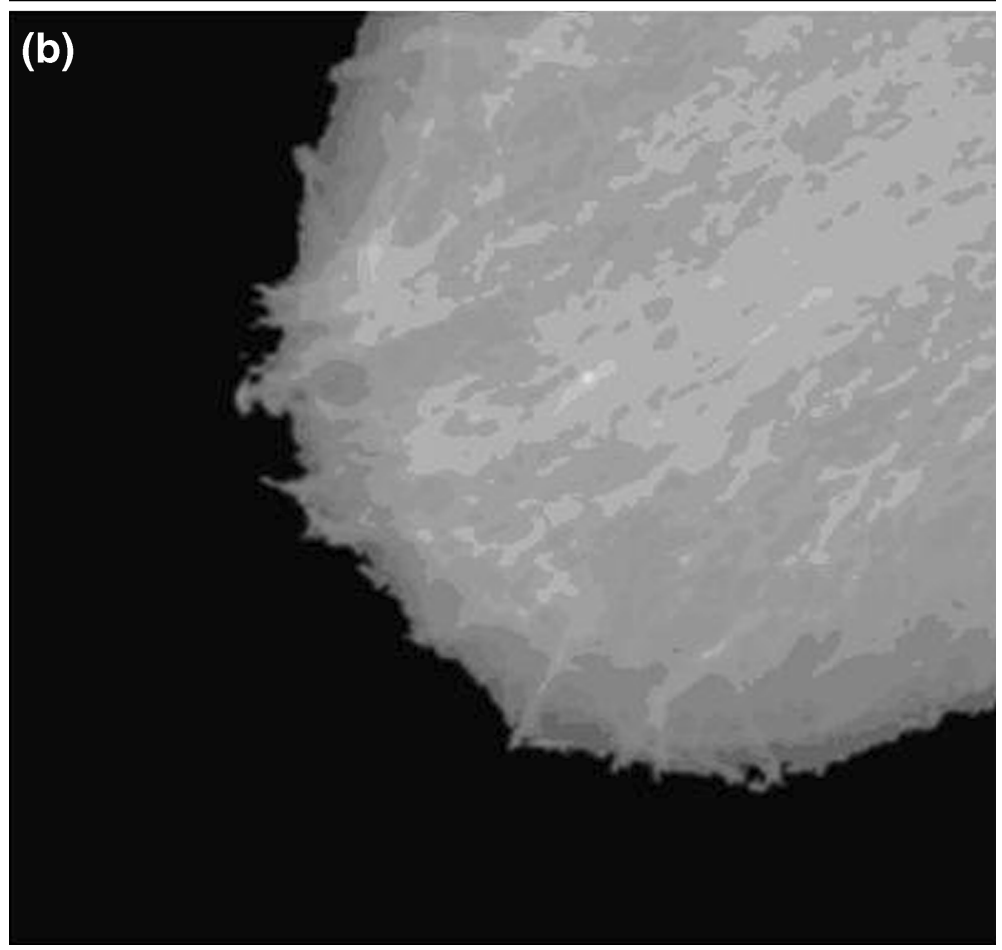

Figure 2. (a). Original digitized mammogram. (b). Mammogram after plain thresholding. 
3.1b Neuron-based thresholding: To filter out the candidate pixels further, a novel approach of performing a second thresholding operation is proposed in this work. The decision of this second threshold method is crucial. If we take a simple average of the gray level intensities of all the pixels, it would not be an unbiased estimator as the pixels with high intensity are small in number.

We therefore, suggest an approach to generate the optimum set of seed points which represent the intensity clusters present in the image. The popular random K-means clustering algorithm has two main disadvantages - the seed points may not necessarily be the best and the number of clusters may not be optimum. An approach proposed by Litinskii (no date) has been used in this work for clustering in the image processing domain. Salient points of the algorithm are presented here.

The seed points for clustering are obtained using a neuron-based procedure with lateral inhibition. Each image point is considered to be a potential seed point and a neuron is assigned to it. Initial activities are assigned to these neurons. Activity is a measure of the distinctness of the particular point. The activities are then updated in corresponding iterations using the transmitting process. The method is explained below.

Let,

$\mathrm{I}_{i}=$ Gray level intensity of the $\mathrm{i}^{\text {th }}$ image point.

$\mathrm{D}_{i j}=$ Intensity distance between the $\mathrm{i}^{\text {th }}$ and the $\mathrm{j}^{\text {th }}$ image point.

$\mathrm{w}_{i j}=$ Connection weight between the $\mathrm{i}^{\text {th }}$ and the $\mathrm{j}^{\text {th }}$ neuron.

$\mathrm{t}=$ Interaction threshold.

$\mathrm{S}_{i}(\mathrm{t})=$ Activity of the $\mathrm{i}^{\text {th }}$ neuron in the $\mathrm{t}^{\text {th }}$ iteration.

$\alpha=$ Transmitting speed.

The various quantities mentioned above are calculated and updated using the gray level intensities of the pixels according to the equations shown in table 1 .

From the above equations it is easy to see that a neuron with large initial activity 'takes away' activities from neurons with whom it interacts. The activities of these surrounding neurons decrease steadily. Finally, a situation will arise when only the non-interacting neurons far from each other with non-zero activities will remain. Subsequent transmitting is impossible and the procedure stops. These image points with non-zero activities are the optimum seed points. The results of the clustering procedure do not depend on the starting conditions. The algorithm is known to give the optimum number of clusters which are actually present in the image.

Table 1. Equations for image clustering.

\begin{tabular}{ll}
\hline No. & Equation \\
\hline 1 & $D_{i j}=\left|I_{i}-I_{j}\right|$ \\
2 & $w_{i j}=\left\{\begin{array}{c}\frac{T^{2}}{D_{i j}^{2}+T^{2}} \\
0 \forall D_{i j}>T\end{array}\right.$ \\
3 & $S_{i}(0)=\sum_{j=1}^{N} w_{i j} \geq 1$ \\
4 & $S_{i}(t+1)=S_{i}(t)+\alpha \sum_{j=1}^{N} w_{i j}\left(S_{i}(t)-S_{j}(t)\right)$ \\
5 & $S_{i}(t)<0 \Rightarrow S_{i}(t)=0$ \\
\hline
\end{tabular}




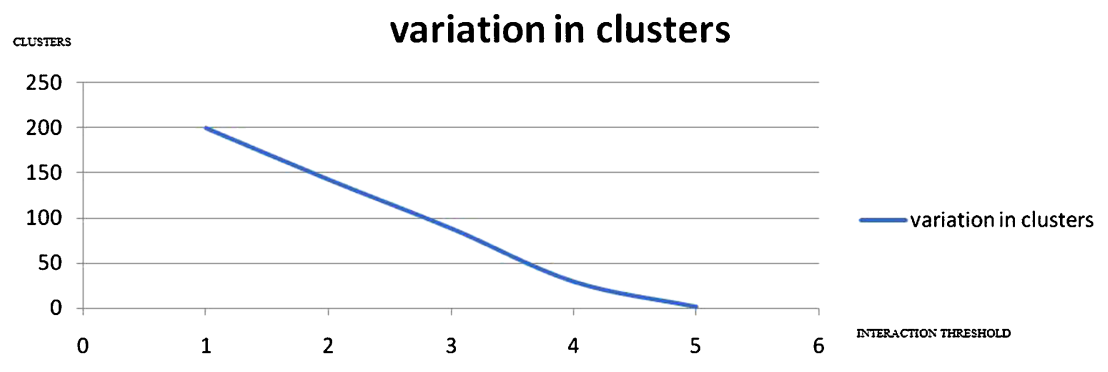

Figure 3. Variation in number of clusters with threshold parameter.

The above algorithm is very sensitive to the choice of the interaction threshold ' $t$ '. If $\mathrm{t}=0$, then not a single neuron interacts with each other. The number of seed points will then be equal to the number of image points. On the other hand, if $t$ is very large, then all the neurons will interact with each other and only one cluster will remain at the end of the transmitting process. In the present work, extensive study was carried out on the performance of the algorithm by taking several images and varying the interaction threshold factor.

Figure 3 shows how the numbers of clusters vary with the choice of $t$. It was observed that on increasing the value of $t$ to 5 , the clusters would collapse. This is due to the fact that the gray level intensities in a typical digitized mammogram are very close to each other. These studies showed an optimum value of $t=3$.This optimum value was used further in our work. Once the optimized set of seed points is obtained for a given image, we take a simple average of the gray level intensities of these points. This average serves as the second threshold for filtering the image.

Figure 4a shows the various intensity clusters formed in the image. The threshold in this case was calculated to be 158 . Figure $4 \mathrm{~b}$ shows the image after the second thresholding operation. The number of candidate pixels got reduced from 140026 in figure $2 \mathrm{~b}$ to 87814 in figure $4 \mathrm{~b}$. It is seen that after passing through the pre-processing module, the candidate pixels reduced to $35 \%$ of the original number present in the image.

\subsection{Neural network classifier}

In order to classify a chosen pixel to be a candidate (affected), a neural network based classifier has been developed in this work. A back propagation neural network (BPNN) architecture using the supervised learning paradigm has been used.

Figure 5 shows the 29-N-1 neural network architecture used in the present work. The input vector of the artificial neural network comprises of a set of 29 statistical feature parameters computed from the digital images ( $\mathrm{Pal}$ et al 2008). These are listed in table 2. The output of the ANN is a single neuron which can have the value of $0 / 1$. The chosen pixel in the image will be processed to compute the 29 parameters (input vector) which will be processed by the trained ANN to give output to classify it as normal or affected.

The ANN configuration was trained by using several images (normal and affected). The affected ones were identified by expert radiologists and formed the training sets for the ANN Various BPNN architectures with one and two hidden layers and of varying number of neurons in the hidden layer were tried out in MATLAB 7.0. The tan sigmoid activation function was used for the hidden layer and the log sigmoid activation function was used for the output layer. The scaled conjugate gradient descent algorithm was used for training with 10000 epochs. 

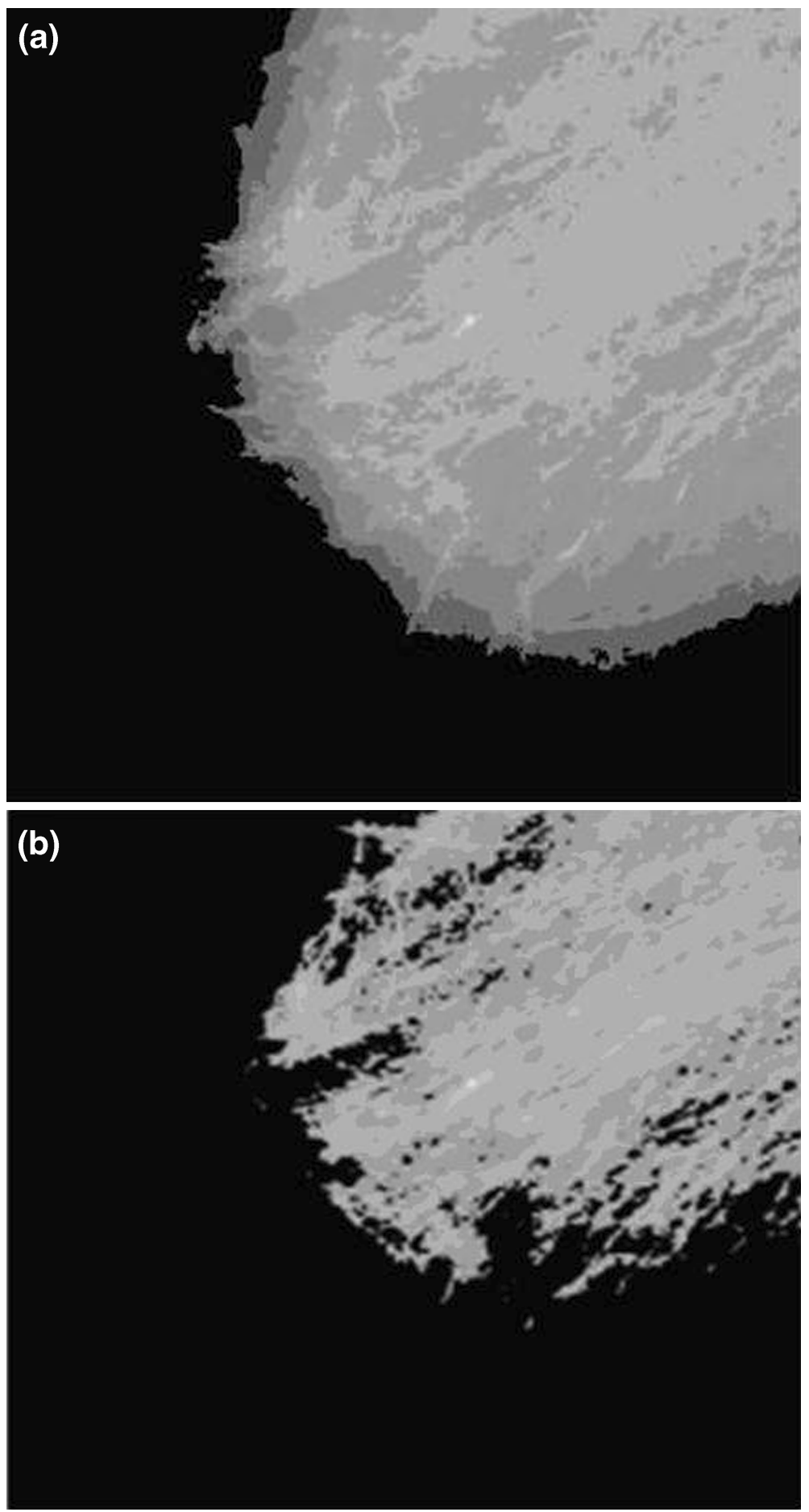

Figure 4. (a). Formation of clusters in the intensity domain. (b). Image after neuron based thresholding. 


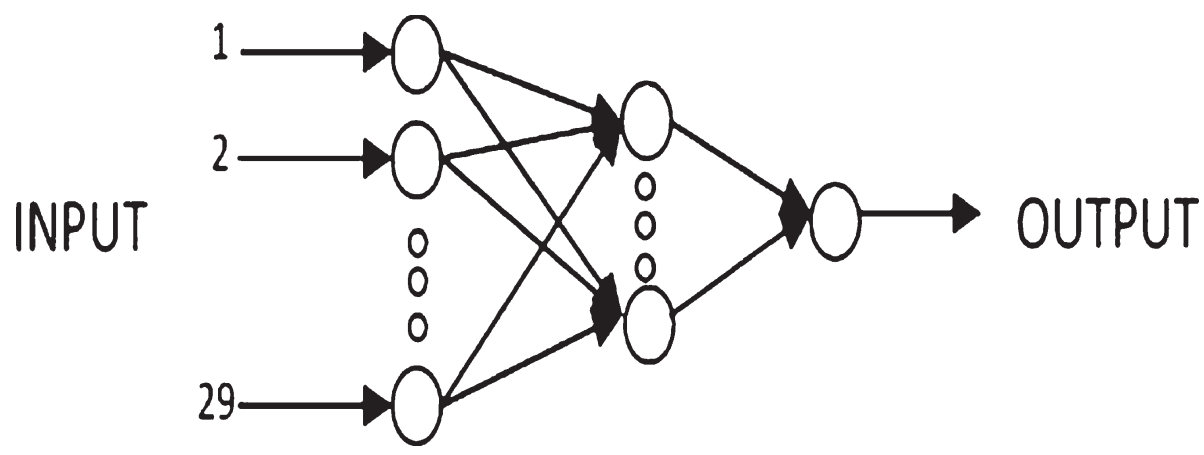

Figure 5. Single hidden layer neural network architecture.

Table 2 shows the various sizes of the square windows used for the calculation of the input vector parameters which are indicated in respective columns. An extensive study was made on the available images to see the effectiveness of each parameter in classification and if some of them could be dropped, especially those parameters which are repeated across different window sizes. It was found that for robust and consistent classification, the chosen feature set of 29 parameters is optimum and essential.

The 29 parameters are calculated for each image pixel. These are then input to a neural network which classifies each image pixel as affected or normal. A training set comprising of 1950 data points was used out of which 128 were affected points. The small ratio of affected to unaffected points was used keeping in mind the fact that in reality there will be very few affected points in an image. This data set was generated in consultation with expert radiologists, by manually selecting the affected and unaffected points. The data set was limited due to lack of availability of mammograms showing microcalcifications as diagnosis in early stages is not very common in India. All the available images and data were used for training and testing.

The neural network was tested on available affected images in the testing set. These images were not used during the training process. The trained system was able to correctly identify the affected pixels. Figure 6a shows the output of the neural network for the image under consideration. The pixels identified by the neural network as affected have been highlighted (gray level intensity of 255) for illustration. The neural network identified 3031 affected points out of the

Table 2. Statistical feature parameters.

\begin{tabular}{llll}
\hline $3 \times 3$ & $5 \times 5$ & $7 \times 7$ & $9 \times 9$ \\
\hline Edge magnitude & Standard deviation & Standard deviation & Standard deviation \\
Edge direction & Moment & Busyness & Moment \\
Standard deviation & Point mask & Potential & Energy variance \\
Moment & Busyness & Kurtosis & Point mask \\
Point mask & Entropy & & Busyness \\
Busyness & Skewness & & Potential \\
Potential & Kurtosis & Edge strength \\
Skewness & & Kurtosis \\
Kurtosis & & Cost function \\
\hline
\end{tabular}



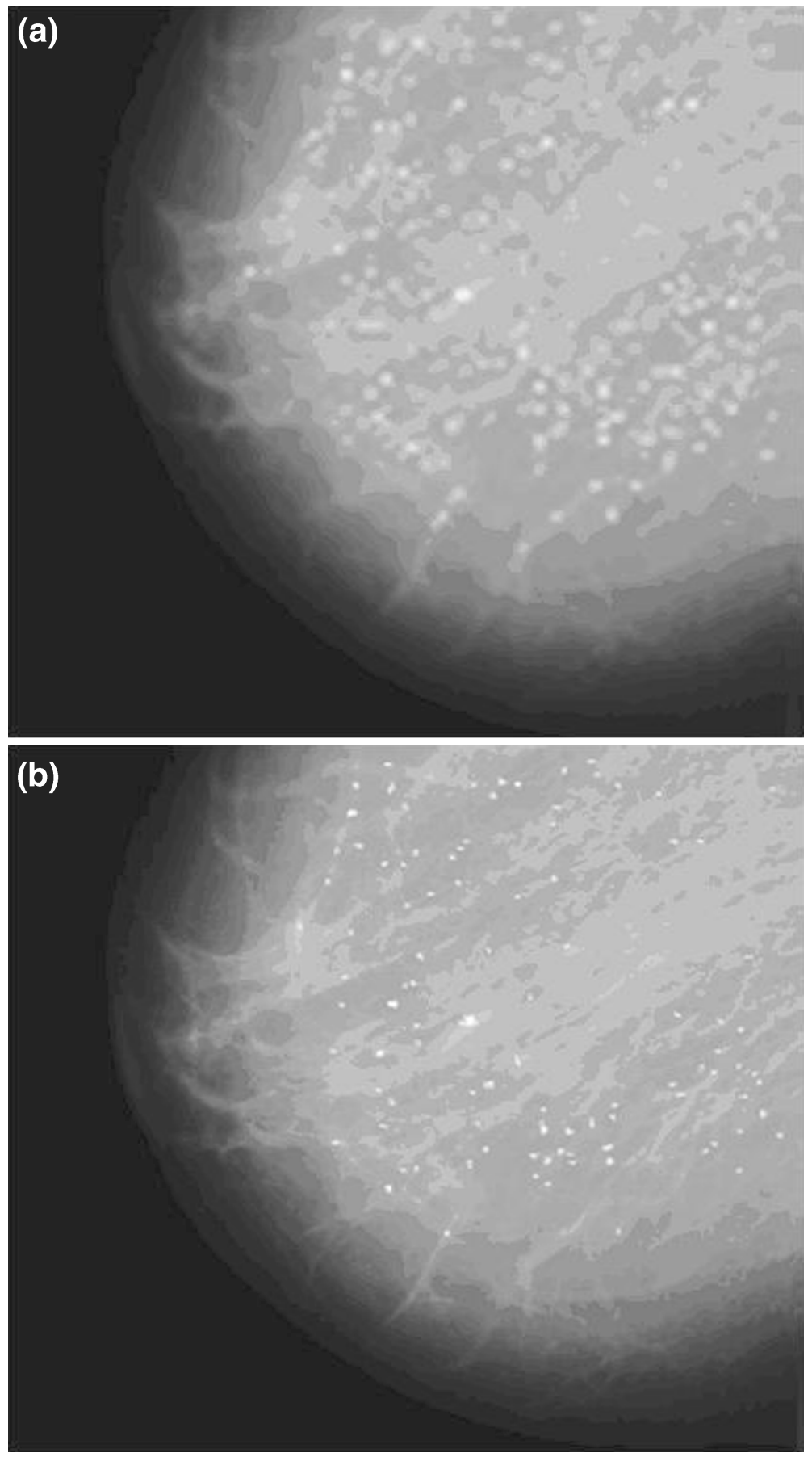

Figure 6. (a). Image after the neural network processing. (b). Connected clusters identified after CCA algorithm. 
87814 candidate pixels. These affected pixels (regions) were confirmed by the expert radiologists as a validity check on the accuracy of prediction by our system.

\subsection{Elimination of false positive}

The output produced by the neural network classifier often consists of a lot of stray points which are not in fact, the microcalcifications. These false positives are eliminated using the algorithms developed in the following modules.

3.3a CCA algorithm: Microcalcifications occur in a clustered form. It was therefore, thought appropriate to compute the connectivity between the pixels which might provide a basis for identification. We calculate the total size of each positive point and its connected components in order to eliminate the ones with connectivity index less than a pre-decided threshold. For this purpose, the connected component analysis (CCA) algorithm proposed by Pal et al (2008) was used. A $3 \times 3$ window was used to find the size of the connected component.

The CCA algorithm scans the pixels to compute the connectivity in the domain. The window is placed across each pixel suggested by the neural network. The pixels are scanned from top to bottom and from left to right and each pixel connected to the centre pixel are labelled. After labelling all the pixels, a second scan is run to clean up and give each component in the image a unique label. Next the number of connected components having specific labels is counted to get the size of each connected component. Component with connected pixels less than or equal to 5 is assumed to be a false positive and removed from further consideration. Extensive experimentation on the available images was done to arrive at the connectivity index of 5 used in this work. Figure $6 \mathrm{~b}$ shows the elimination of false positives from the image output by the neural network classifier in the previous stage.

3.3b Removal of elongated components: The breast tissue contains a lot of elongated high intensity components which feature as false positives in the output of the neural network classifier. These false positives can be eliminated by shape analysis of the clusters e.g., by taking a ratio of the area to perimeter of the cluster. The elongated structures will be less dense and will have a similar area and perimeter value whereas the actual microcalcifications will have a higher area as compared to the perimeter. Shape analysis of this type is a common approach adopted by many researchers in automatic detection. Jiang et al (2007) has used shape features like aspect ratio, compactness and elongation of suspected region as shape features.

In the present work, we have used the number of connected pixels as calculated in the previous section as a measure of the area of the component. For calculating the boundary pixels, we check for 'eight connectivity' using a $3 \times 3$ window. If all the eight connected pixels are not affected in the neural network output, then it is a boundary pixel. We retain all the components having area versus perimeter ratio greater than 1 . This value has been arrived at after rigorous testing of the available data set. It can be further optimized for better accuracy if more data is available. Figure $7 \mathrm{a}$ shows the image after further cleaning by the removal of elongated components.

3.3c Detection of microcalcification: Microcalcifications occur as clusters whereas the false positives are sparsely distributed. We therefore, check for aggregation of the positive points which have remained subsequent to the removal of false positives by the CCA and elongated component elimination modules. For this purpose we take a filter of size $21 \times 21$ with all entries 


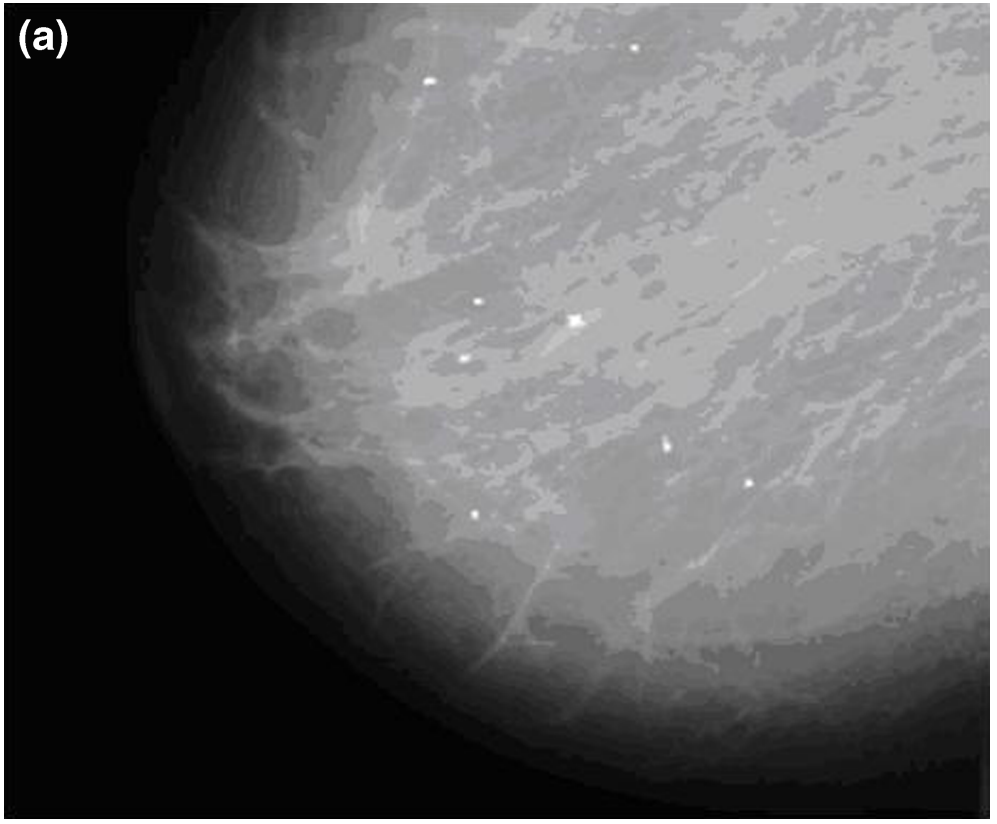

(b)

Figure 7. (a). Mammogram with elongated components removed. (b). Prompt for detection of microcalcification. 
1. This window size was arrived after repeated testing and optimization of the system on the available data set. This filter is moved over each of the affected pixels in the binarized image matrix and a dot product is taken in order to check for a cluster of positive points. All clusters of size less than 20 and greater than 70 are eliminated from further consideration while the rest are identified and marked (encircled) as potential areas for the presence of microcalcification. The above values [20-70] were arrived at after extensively studying the size of positive clusters in the images available. Figure $7 \mathrm{~b}$ shows the microcalcification identified after the cluster check. The size of the cluster is found to be 35 pixels. The system took about 160 seconds for the detection.

\subsection{Diagnosis}

The suspected area on the image is marked with a red circle (figure $7 \mathrm{~b}$ ). The radiologist can further critically study the images for final diagnosis.
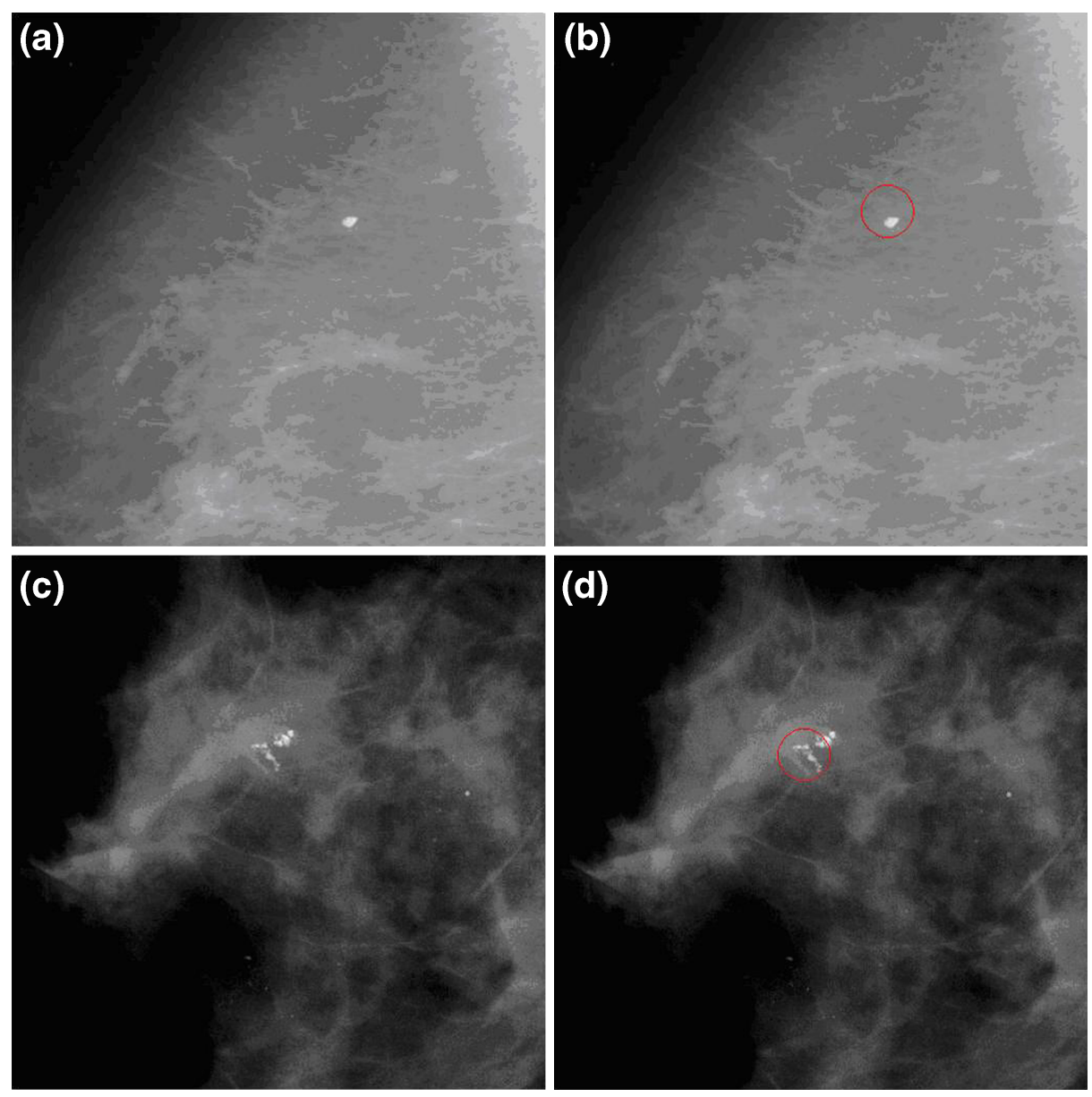

Figure 8. (a) and (c). Original digitized mammograms. (b) and (d). Mammograms with detection prompts. 


\section{Implementation and testing}

The system discussed above was developed in Visual Basic 6.0 and tested on a Intel Core 2 Duo machine with 1 GB RAM. The system was rigorously tested for the available mammogram images. Shown below is the diagnosis for two typical abnormal (affected) images.

The system successfully detected the microcalcification for the images shown in figures $8 \mathrm{a}$ and c. The second threshold values (Sec. 3.1b) were 150 and 166, the cluster of positive points detected was 32 and 30 . The system took 64 and 16 seconds respectively for detection. The detection prompts are shown in figures $8 \mathrm{~b}$ and $\mathrm{d}$ respectively.

\section{Conclusions}

An intelligent system has been developed and implemented for the detection of the calcified area from digital mammograms using artificial neural network techniques. The system was tuned to eliminate false positives using CCA and elongated component algorithms. The developed system was rigorously tested on available images and was found to be robust, efficient and accurate. The system works reasonably fast on any PC and does not need any proprietary software. It can thus, be conveniently used for real life applications. The output image with prompts generated by the system can form an important input to a radiologist for the final diagnosis. Future improvements in the system would include using a larger training set for better accuracy and the optimization of the system parameters. Classification of the detected region as benign or malignant will be a challenging area for future research.

\section{References}

Delogu P, Fantacci M, Kasae P, Retico A 2007 Charaterization of mammographic masses using a gradientbased segmentation algorithm and a neural classifier. Comput. Biol. Med. 37: 1479-1491

Essex University, http://peipa.essex.ac.uk/info/mias.html viewed 19th January 2009

Halkiotis S, Botsis T, Rangoussi M 2007 Automatic detection of clustered microcalcifications in digital mammograms using mathematical morphology and neural networks. Signal Process. 87: 1559-1568

Jemal A, Clegg L, Ward E, Ries L, Wu X, Jamison P, Wingo P, Howe H, Anderson R, Edwards B 2004 Annual report to the nation on the status of cancer, 1975-2001, with a special feature regarding survival. Cancer 101(1): 3-27

Jiang J, Yao B, Wason A 2007 A genetic algorithm design for micro-calcification detection and classification in digital mammograms. Comput. Med. Imaging Graph. 31: 49-61

Laine A, Schuler S, Fan J, Huda W 1994 Mammographic feature enhancement by multiscale analysis. IEEE Trans. Med. Imaging 13(4): 725-740

Litinskii L, Romanov D Neural network clustering based on distances between objects. Russian Academy of Sciences, http://arxiv.org/ftp/cs/papers/0608/0608115.pdf. viewed 24th April 2009

National Cancer Registry Project 2001 Consolidated report of the population based cancer registries 19901996, ICMR, New Delhi

Oh W V, Kim K G, Kim Y J, Kan H S, Ro J S, Moon W K 2009 Detection of microcalcifications in digital mammograms using Foveal method. J. Kor. Soc. Med. Informatics 15(1): 165-172

Pal N, Bhowmick B, Patel S, Pal S, Das J 2008 A multi-stage neural network aided system for detection of microcalcifications in digitized mammograms. Neurocomputing 71: 2625-2634

Parkin D, Bray F, Ferlay J, Pisani P 2001 Estimating the world cancer burden: globocon 2000. Int. J. Cancer 94: 153-156

Rangayyan R, Ayres F, Desautels L 2007 A review of computer aided diagnosis of breast cancer: Toward the detection of subtle signs. J. Franklin Inst. 344: 312-348 
Stojic T, Reljin I, Reljin B 2006 Adaptation of multifractal analysis to segmentation of microcalcifications in digital mammograms. Physica A367: 494-508

Wei J, Chan H P, Sahiner B, Zhou C, Hadijiski L M, Roubidox M A, Helvie M A 2009 Computer-aided detection of breast masses on mammograms: Dual system approach with two-view analysis. Med. Phys. 36: $4451-4460$ 